\title{
ASYMPTOTIC BEHAVIOR OF SOLUTIONS TO THE HELMHOLTZ EQUATIONS WITH SIGN CHANGING COEFFICIENTS
}

\author{
HOAI-MINH NGUYEN
}

\begin{abstract}
This paper is devoted to the study of the behavior of the unique solution $u_{\delta} \in H_{0}^{1}(\Omega)$, as $\delta \rightarrow 0$, to the equation

$$
\operatorname{div}\left(s_{\delta} A \nabla u_{\delta}\right)+k^{2} s_{0} \Sigma u_{\delta}=s_{0} f \text { in } \Omega,
$$

where $\Omega$ is a smooth connected bounded open subset of $\mathbb{R}^{d}$ with $d=2$ or $3, f \in L^{2}(\Omega), k$ is a non-negative constant, $A$ is a uniformly elliptic matrixvalued function, $\Sigma$ is a real function bounded above and below by positive constants, and $s_{\delta}$ is a complex function whose real part takes the values 1 and -1 and whose imaginary part is positive and converges to 0 as $\delta$ goes to 0 . This is motivated from a result of Nicorovici, McPhedran, and Milton; another motivation is the concept of complementary media. After introducing the reflecting complementary media, complementary media generated by reflections, we characterize $f$ for which $\left\|u_{\delta}\right\|_{H^{1}(\Omega)}$ remains bounded as $\delta$ goes to 0 . For such an $f$, we also show that $u_{\delta}$ converges weakly in $H^{1}(\Omega)$ and provide a formula to compute the limit.
\end{abstract}

\section{INTRODUCTION}

Negative index materials (NIMs) were first investigated theoretically by Veselago in 22] and were innovated by Pendry in [15]. The existence of such materials was confirmed by Shelby, Smith, and Schultz in [20] (see also [21]). Cloaking space and illusion optics using NIMs were discussed in [7,8] (see also [14]) based on the concept of complementary and in $[2,3,5,6,11,14$ based on the anomalous localized resonance. Perfect lens using NIMs was studied in 13, 14, 16, 19,

The first motivation for this work comes from the following two-dimensional result of Nicorovici et al. in 14. Let $0<r_{1}<r_{2}<R$, and $f \in L^{2}\left(B_{R}\right)$. Here and in what follows, for $r>0, B_{r}$ denotes the ball centered at 0 of radius $r$. Set $r_{3}=r_{2}^{2} / r_{1}$. Assume that $R>r_{3}$ and $\operatorname{supp} f \cap\left\{x|x|<r_{3}\right\}=\varnothing$. Let $u_{\delta} \in H_{0}^{1}\left(B_{R}\right)$ be the unique solution to the equation

$$
\operatorname{div}\left(\varepsilon_{\delta} \nabla u_{\delta}\right)=f \text { in } B_{R} .
$$

Here

where

$$
\varepsilon_{\delta}=\varepsilon+i 1_{r_{1}<|x|<r_{2}},
$$

$$
\varepsilon(x)=\left\{\begin{array}{cl}
-1 & \text { if } r_{1}<|x|<r_{2}, \\
1 & \text { otherwise }
\end{array}\right.
$$

Received by the editors September 23, 2013.

2010 Mathematics Subject Classification. Primary 35B40, 35Q60; Secondary 78A40, 78M35.

This research was supported by NSF grant DMS-1201370 and by the Alfred P. Sloan Foundation. 
Physically, the imaginary part of $\varepsilon_{\delta}$ is the loss of the medium. It is shown in [14] by separation of variables that

$$
u_{\delta} \rightarrow \mathcal{U} \text { for }|x|>r_{3},
$$

where $\mathcal{U} \in H_{0}^{1}\left(B_{R}\right)$ is the unique solution to the equation

$$
\Delta \mathcal{U}=f \text { in } B_{R} .
$$

The surprising fact in this result is that (1.2) holds for any $f$ with $\operatorname{supp} f \cap B_{r_{3}}=\varnothing$. From (1.2), one might say that the region $\left\{r_{2}<|x|<r_{3}\right\}$ is canceled by the one in $\left\{r_{1}<|x|<r_{2}\right\}$ and the total system is effectively equal to the free space; invisibility is achieved.

The following questions naturally arise:

- What happens if $\operatorname{supp} f \cap B_{r_{3}} \neq \varnothing$ ?

- Is the radial symmetry necessary? If not, what are conditions on $\varepsilon$ ?

- What happens in the finite frequency regime?

- Do similar phenomena hold in three dimensions? If yes, under which conditions?

Another motivation for this work is the concept of complementary media which was suggested in [7,18, 19] (see also [14,15]). This concept has played an important role in the study of NIMs and its applications such as cloaking, perfect lens, and illusion optics (see [7,8,12,15, 19]). Although many examples have been suggested, this concept has not been defined in a precise manner. A common point in examples studied is

$$
F_{*} a=-b \text { in } D_{2},
$$

for some diffeomorphism $F: D_{1} \rightarrow D_{2}$ if a matrix $a$ defined in a region $D_{1}$ is complementary to a matrix $b$ defined in a region $D_{2}$. Here

$$
F_{*} a(y)=\frac{D F(x) a(x) D F^{T}(x)}{J(x)}, \text { where } x=F^{-1}(y) \text { and } J(x)=|\operatorname{det} D F(x)| .
$$

It is easy to verify in two dimensions that if $F:\left\{r_{1}<|x|<r_{2}\right\} \rightarrow\left\{r_{2}<|x|<r_{3}\right\}$ with $r_{3}=r_{2}^{2} / r_{1}$ defined by $F(x)=r_{2}^{2} x /|x|^{2}$, then $F$ is a diffeomorphism and

$$
F *(-I)=-I \text { in }\left\{r_{2}<|x|<r_{3}\right\} .
$$

In other words, the medium $-I$ in $\left\{r_{1}<|x|<r_{2}\right\}$ is complementary to the medium $I$ in $\left\{r_{2}<|x|<r_{3}\right\}$ : complementary media appears in the setting of Nocorovici et al.

In this paper, we address the above questions. To this end, we first introduce the notion of reflecting complementary media. Similar phenomena as in (1.2) take place for media that inherits this property in the quasistatic and the finite frequency regimes. Two media (two matrices in two regions in the quasistatic regime) are called reflecting complementary if they are complementary and the complementary is generated by a reflection which satisfies some mild conditions. The motivation for the definition of this notion comes from the reflection technique used in a heuristic argument for (1.2) in Section 2.1. We then establish results in the spirit of (1.2) for media of this property. The analysis again has roots in the heuristic argument. The key to the analysis is the derivation of two Cauchy problems for elliptic equations by the reflection technique using the reflecting complementary property. Concerning the analysis, we characterize $f$ (based on the compatibility condition in Definition 2) 
for which $\left\|u_{\delta}\right\|_{H^{1}}$ remains bounded as $\delta \rightarrow 0_{+}$; moreover, we show that for such a function $f$, the limit of $u_{\delta}$ exists as $\delta \rightarrow 0_{+}$(Theorem 1). We also provide a formula to compute the limit which involves only the solutions of standard elliptic equations (no sign changing coefficients), and show that the limit has properties in the spirit of (1.2) (Theorem 2). To our knowledge, the results presented in this paper are new even in the $2 \mathrm{~d}$-quasistatic regime.

The use of reflections to study NIMs has been considered previously in [12. However, there is a big difference between the use of reflections in [12] and in this paper. In [12, the authors used reflections as a change of variables to obtain a new simple setting from an old more complicated one and hence the analysis of the old problem becomes simpler. In this paper, we use reflections to derive the two Cauchy problems. This derivation essentially makes use of the complementary property of media. The global or non-global existence of the solutions to these Cauchy problems will determine the boundedness or unboundedness of the $H^{1}$-norm of the solutions.

The goals and the setting of this paper are different from Ammari et al.'s in 2 and Kohn et al.'s in 6. In this paper, we introduce the concept of reflecting complementary media and we study the boundedness of $\left\|u_{\delta}\right\|_{H^{1}}$ and the limit of $u_{\delta}$ in the whole domain as $\delta \rightarrow 0$ in the quasistatic and the finite frequency regimes. In [2, 6], the authors investigate the unboundedness of $\delta^{1 / 2}\left\|u_{\delta}\right\|_{H^{1}}$ for piecewise constant media (up to a diffeomorphism in [6]) in the quasistatic case. It is clear that the boundedness of $\left\|u_{\delta}\right\|_{H^{1}}$ implies the boundedness of $\delta^{1 / 2}\left\|u_{\delta}\right\|_{H^{1}}$ and the unboundedness of $\delta^{1 / 2}\left\|u_{\delta}\right\|_{H^{1}}$ implies the unboundedness of $\left\|u_{\delta}\right\|_{H^{1}}$. The media considered in 6] are of the reflecting complementary property; however the ones in [2] are, in general, not (the radial setting considered in [2, Section 5] is an exclusion). In [2], the authors also deal with the boundedness of $u_{\delta}$ in some region. To make use of their results mentioned above, one needs detailed information on the spectral properties of certain boundary integral operators. This information is difficult to come by in general. The method in this paper is different from and more elementary than the spectral one in [2] and the variational one in [6].

The method in this paper is used in [9] and developed in [10]. In [9] the authors study the complete resonance and localized resonance in plasmonic structures whereas in [10] the author investigates the cloaking via complementary media.

Our paper is organized as follows. Section 2 contains two subsections devoted to the concept of reflecting complementary media. In the first subsection, we present the heuristic argument to obtain (1.2) and to motivate the definition of this concept. The second subsection is devoted to the definition. In Section 3, we state and prove properties on the reflecting complementary media. More precisely, we state and prove Theorems 1 and 2 and present their two corollaries.

\section{Reflecting COMPlEMEntary Media}

In this section, we introduce the notion of reflecting complementary media. To motivate the definition, we first present a heuristic argument, in Section 2.1, to obtain (1.2) based on the reflecting technique. The definition of reflecting complementary media is introduced in Section 2 .

2.1. Motivation - a heuristic argument for (1.2). In this section, we present a heuristic (elementary) argument to obtain (1.2). This argument motivates not only the notion of the reflecting complementary media but also the analysis in Section 3 . 
In this section, we assume that $u_{\delta} \rightarrow u \in H^{1}\left(B_{R}\right)$. It follows that $u \in H_{0}^{1}\left(B_{R}\right)$ is a solution to the equation

$$
\operatorname{div}(\varepsilon \nabla u)=f \text { in } B_{R}
$$

Let $F$ defined in $\left\{|x|<r_{2}\right\}$ be the Kelvin transform w.r.t. $\partial B_{r_{2}}$, i.e.,

$$
F(x)=\frac{r_{2}^{2}}{|x|^{2}} x \text { for }|x|<r_{2} .
$$

Let $u_{1}$ defined in $\left\{|x|>r_{2}\right\}$ be the Kelvin transform $F$ of $u$, i.e.,

$$
u_{1}(x)=u \circ F^{-1}(x) \quad \text { for }|x|>r_{2} .
$$

Then, by the transmission condition on $\partial B_{r_{2}}$, we have

$$
u_{1}=u \quad \text { and }\left.\quad \partial_{r} u_{1}\right|_{r \rightarrow r_{2+}}=\left.\partial_{r} u\right|_{r \rightarrow r_{2+}} \text { for }|x|=r_{2} .
$$

Since $F$ is a Kelvin transform and supp $f \cap\left\{|x|<r_{3}\right\}=\varnothing$, it follows that

$$
\operatorname{div}\left(\hat{\varepsilon} \nabla u_{1}\right)=0 \text { for }|x|>r_{2},
$$

where

$$
\hat{\varepsilon}(x)=\left\{\begin{array}{cl}
1 & \text { if } r_{2}<|x|<r_{3}, \\
-1 & \text { if }|x|>r_{3} .
\end{array}\right.
$$

(Note that $F$ transforms $\partial B_{r_{1}}$ into $\partial B_{r_{3}}$.) By the unique continuation principle, we have

$$
u_{1}=u \text { in }\left\{r_{2}<|x|<r_{3}\right\} .
$$

Let $G$ defined in $\left\{|x|>r_{3}\right\}$ be the Kelvin transform w.r.t. $\partial B_{r_{3}}$, i.e.,

$$
G(x)=\frac{r_{3}^{2}}{|x|^{2}} x \text { for }|x|>r_{3} .
$$

Define $u_{2}$ in $\left\{|x|<r_{3}\right\}$, the Kelvin transform $G$ of $u_{1}$, as

$$
u_{2}(x)=u_{1} \circ G^{-1}(x) \quad \text { for }|x|<r_{3} .
$$

Similar to (2.2), we have

$$
u_{2}=u_{1} \quad \text { and }\left.\quad \partial_{r} u_{2}\right|_{r \rightarrow r_{3-}}=\left.\partial_{r} u_{1}\right|_{r \rightarrow r_{3-}} \quad \text { for }|x|=r_{3} .
$$

It follows from (2.5) that

$$
u_{2}=u \quad \text { and }\left.\quad \partial_{r} u_{2}\right|_{r \rightarrow r_{3-}}=\partial_{r} u \quad \text { for }|x|=r_{3} .
$$

We also have

$$
\Delta u_{2}=0 \text { in }|x|<r_{3},
$$

by the property of the Kelvin transforms. Define $\mathcal{U}$ by

$$
\mathcal{U}(x)=\left\{\begin{array}{cc}
u(x) & \text { if }|x|>r_{3}, \\
u_{2}(x) & \text { if }|x|<r_{3} .
\end{array}\right.
$$

Since $\Delta u=f$ for $|x|>r_{3}$, it follows from (2.8) and (2.9) that

$$
\Delta \mathcal{U}=f \text { in } B_{R} .
$$

Therefore, we obtain (1.2). 
2.2. Reflecting complementary media. In this section, we introduce the notion of reflecting complementary media. Let $\Omega_{1} \subset \subset \Omega_{2} \subset \subset \Omega_{3} \subset \subset \Omega$ be smooth connected bounded open subsets of $\mathbb{R}^{d}(d=2,3)$.

Let $A$ be a measurable matrix function and let $\Sigma$ be a measurable real function defined in $\Omega$ such that

$$
\frac{1}{\Lambda}|\xi|^{2} \leq\langle A(x) \xi, \xi\rangle \leq \Lambda|\xi|^{2} \quad \forall \xi \in \mathbb{R}^{d},
$$

for a.e. $x \in \Omega$ and for some $0<\Lambda<+\infty$, and

$$
0<\underset{\Omega}{\operatorname{ess} \inf } \Sigma \leq \text { ess } \sup \Sigma<+\infty .
$$

Set

$$
s_{\delta}(x)=\left\{\begin{array}{cl}
-1+i \delta & \text { if } x \in \Omega_{2} \backslash \Omega_{1}, \\
1 & \text { otherwise. }
\end{array}\right.
$$

We are later interested in the behavior of the unique solution $u_{\delta} \in H_{0}^{1}(\Omega)$ to the equation

$$
\operatorname{div}\left(s_{\delta} A \nabla u_{\delta}\right)+k^{2} s_{0} \Sigma u_{\delta}=s_{0} f \text { in } \Omega,
$$

as $\delta \rightarrow 0$.

We are ready to give

Definition 1 (Reflecting complementary media). The media $(A, \Sigma)$ in $\Omega_{3} \backslash \Omega_{2}$ and $(-A,-\Sigma)$ in $\Omega_{2} \backslash \Omega_{1}$ are said to be reflecting complementary if there exists a diffeomorphism $F: \Omega_{2} \backslash \bar{\Omega}_{1} \rightarrow \Omega_{3} \backslash \bar{\Omega}_{2}$ such that

$$
F_{*} A(x)=A(x), \quad F_{*} \Sigma(x)=\Sigma(x) \text { for } x \in \Omega_{3} \backslash \bar{\Omega}_{2},
$$

$$
F(x)=x \text { on } \partial \Omega_{2},
$$

and the following two conditions hold:

1) There exists a diffeomorphism extension of $F$, which is still denoted by $F$, from $\Omega_{2} \backslash\left\{x_{1}\right\} \rightarrow \Omega_{4} \backslash \bar{\Omega}_{2}$ for some $x_{1} \in \Omega_{1}$ and some smooth open subset $\Omega_{4}$ of $\mathbb{R}^{d}$ with $\Omega_{3} \subset \Omega_{4}$.

2) There exists a diffeomorphism $G: \Omega_{4} \backslash \bar{\Omega}_{3} \rightarrow \Omega_{3} \backslash x_{2}$ for some $x_{2} \in \Omega_{3}$ such that 1

$$
G(x)=x \text { on } \partial \Omega_{3}
$$

and

$$
G \circ F: \Omega_{1} \rightarrow \Omega_{3} \text { is a diffeomorphism if one sets } G \circ F\left(x_{1}\right)=x_{2} .
$$

Here and in what follows, we use the standard notation:

$$
\mathcal{F}_{*} \mathcal{A}(y)=\frac{D \mathcal{F}(x) \mathcal{A}(x) D \mathcal{F}^{T}(x)}{J(x)}, \quad \mathcal{F}_{*} \Sigma(y)=\frac{\Sigma(x)}{J(x)}, \quad \text { and } \quad \mathcal{F}_{*} f(y)=\frac{f(x)}{J(x)},
$$

where $x=\mathcal{F}^{-1}(y)$ and $J(x)=|\operatorname{det} D \mathcal{F}(x)|$.

Some comments on the definition are:

i) If $k=0$, then the condition on $\Sigma$ is irrelevant in Definition 1 .

\footnotetext{
${ }^{1}$ In (2.16) and 2.17), $F$ and $G$ denote some diffeomorphism extensions of $F$ and $G$ in a neighborhood of $\partial \Omega_{2}$ and of $\partial \Omega_{3}$.
} 
ii) Condition (2.15) implies that $(A, \Sigma)$ in $\Omega_{3} \backslash \Omega_{2}$ and $(-A,-\Sigma)$ in $\Omega_{2} \backslash \Omega_{1}$ are complementary in the usual sense. The term "reflecting" in the definition comes from (2.16) and the assumption $\Omega_{1} \subset \Omega_{2} \subset \Omega_{3}$. Conditions (2.15) and (2.16) are the main assumptions in the definition.

iii) Condition (2.15) makes sure that $u$ (the "solution" for $\delta=0$ ) and $u_{1}:=u \circ F$ satisfy the same equation in $\Omega_{3} \backslash \Omega_{2}$; hence the reflecting technique in Section 2.1 can be used. Conditions (2.16) and (2.17) assure that $u=u_{1}$ on $\partial \Omega_{2}$ and $u_{2}=u_{1}$ on $\partial \Omega_{3}$ where $u_{2}=u_{1} \circ G^{-1}$. Condition (2.18) is a technical one which is required by the proof.

iv) Conditions 1) and 2) in the definition are mild assumptions. Introducing $G$ in the definition makes the analysis more accessible as in Section 2.1 (see the paragraph below (3.14) for other comments on $G$ ).

v) In general, it is difficult to verify whether (2.15) holds for some $F$. In practice, to obtain the reflecting complementary in $\Omega_{2} \backslash \Omega_{1}$ for $(A, \Sigma)$ in $\Omega_{3} \backslash \Omega_{2}$, it suffices to choose a diffeomorphism $F: \Omega_{2} \backslash\left\{x_{1}\right\} \rightarrow \Omega_{4} \backslash \bar{\Omega}_{2}$ for some $x_{1} \in \Omega_{1}$ and for some smooth bounded open subset $\Omega_{4}$ containing $\Omega_{3}$, and define $(-A,-\Sigma)$ in $\Omega_{2} \backslash \Omega_{1}$ by $\left(-F_{*}^{-1} A,-F_{*}^{-1} \Sigma\right)$.

It is clear that the medium $\varepsilon$ given in (2.4) has the reflecting complementary property with $\Omega_{i}=B_{r_{i}}$ (for $i=1,2,3$ ) and $\Omega=B_{R}$ where $r_{4}:=+\infty$, and $F$ and $G$ are the Kelvin transforms w.r.t. $\partial B_{r_{2}}$ and $\partial B_{r_{3}}$, resp.

Remark 1. Concerning reflecting complementary media, the 2d-quasistatic case is quite special in the sense that two constant media (two constant matrices) can be complementary (see the example in the introduction). In fact, in the 2 d-finite frequency case, it seems that there do not exist two constant media (two constant matrices and two constant functions) which are complementary and in the $3 \mathrm{~d}$ case there do not exist two constant media (two constant matrices) which are complementary.

\section{Properties Related to Reflecting complementary media}

3.1. Statement of the results. Let $\Omega_{1} \subset \subset \Omega_{2} \subset \subset \Omega_{3} \subset \subset \Omega$ be smooth connected bounded open subsets of $\mathbb{R}^{d}(d=2,3)$. Let $A$ be a measurable matrix function and let $\Sigma$ be a measurable real function defined in $\Omega$ such that

$$
\frac{1}{\Lambda}|\xi|^{2} \leq\langle A(x) \xi, \xi\rangle \leq \Lambda|\xi|^{2} \quad \forall \xi \in \mathbb{R}^{d}
$$

for a.e. $x \in \Omega$ and for some $0<\Lambda<+\infty$, and

$$
0<\underset{\Omega}{\operatorname{ess} \inf } \Sigma \leq \text { ess } \sup _{\Omega} \Sigma<+\infty .
$$

We will assume that $A$ is piecewise differentiable in $\Omega$ in three dimensions 2

Set

$$
s_{\delta}(x)=\left\{\begin{array}{cl}
-1+i \delta & \text { if } x \in \Omega_{2} \backslash \Omega_{1}, \\
1 & \text { otherwise. }
\end{array}\right.
$$

We are interested in the behavior of the unique solution $u_{\delta} \in H_{0}^{1}(\Omega)$ to the equation

$$
\operatorname{div}\left(s_{\delta} A \nabla u_{\delta}\right)+k^{2} s_{0} \Sigma u_{\delta}=s_{0} f \text { in } \Omega,
$$

as $\delta \rightarrow 0$.

\footnotetext{
${ }^{2}$ This condition is necessary to obtain the uniqueness for the Cauchy problems.
} 
Throughout this section, we will assume that:

systems (3.6) and (3.7) have only zero solutions in $H^{1}\left(\Omega \backslash \bar{\Omega}_{2}\right)$ and $H^{1}(\Omega)$, resp.,

where

$$
\left\{\begin{array}{cl}
\operatorname{div}(A \nabla v)+k^{2} \Sigma v=0 & \text { in } \Omega \backslash \bar{\Omega}_{2}, \\
v=0 & \text { on } \partial \Omega \cup \partial \Omega_{2},
\end{array}\right.
$$

and

$$
\left\{\begin{array}{cl}
\operatorname{div}(\hat{A} \nabla U)+k^{2} \hat{\Sigma} U=0 & \text { in } \Omega \\
U=0 & \text { on } \partial \Omega .
\end{array}\right.
$$

Here $\hat{A}$ and $\hat{\Sigma}$ are defined as

$$
\hat{A}:=\left\{\begin{array}{cl}
A & \text { if } x \in \Omega \backslash \Omega_{3}, \\
G_{*} F_{*} A & \text { if } x \in \Omega_{3},
\end{array} \text { and } \quad \hat{\Sigma}:=\left\{\begin{array}{cl}
\Sigma & \text { if } x \in \Omega \backslash \Omega_{3}, \\
G_{*} F_{*} \Sigma & \text { if } x \in \Omega_{3} .
\end{array}\right.\right.
$$

We also define

$$
\hat{f}:=\left\{\begin{array}{cl}
f & \text { if } x \in \Omega \backslash \Omega_{3}, \\
G_{*} F_{*} f & \text { if } x \in \Omega_{3} .
\end{array}\right.
$$

Remark 2. The well-posedness of (3.6) and (3.7) always holds for $k=0$. In the case $k>0$, if one is interested the corresponding problem on the whole space in which outgoing solutions are considered, the well-posedness assumption is not necessary.

In what follows we assume that $k>0$. The case $k=0$ is similar and even easier to obtain. The first main result in this section is

Theorem 1. Let $d=2,3, \delta>0, f \in L^{2}(\Omega)$ and let $u_{\delta} \in H_{0}^{1}(\Omega)$ be the unique solution to equation (2.14):

$$
\operatorname{div}\left(s_{\delta} A \nabla u_{\delta}\right)+k^{2} s_{0} \Sigma u_{\delta}=s_{0} f \text { in } \Omega .
$$

Assume that the media $(A, \Sigma)$ in $\Omega_{3} \backslash \Omega_{2}$ and $(-A,-\Sigma)$ in $\Omega_{2} \backslash \Omega_{1}$ are reflecting complementary. We have

a) Case 1: $f$ is compatible with the medium. Then $\left(u_{\delta}\right)$ converges weakly in $H^{1}(\Omega)$ and strongly in $L^{2}(\Omega)$ to $u_{0} \in H_{0}^{1}(\Omega)$, the unique solution to the equation

$$
\operatorname{div}\left(s_{0} \nabla u_{0}\right)+k^{2} s_{0} \Sigma u_{0}=s_{0} f \text { in } \Omega,
$$

as $\delta \rightarrow 0$.

b) Case 2: $f$ is not compatible with the medium. We have

$$
\lim _{\delta \rightarrow 0}\left\|u_{\delta}\right\|_{H^{1}(\Omega)}=+\infty .
$$

In the statement of Theorem 1, we use the following

Definition 2 (Compatibility condition). Assume that the media $(A, \Sigma)$ in $\Omega_{3} \backslash \Omega_{2}$ and $(-A,-\Sigma)$ in $\Omega_{2} \backslash \Omega_{1}$ are reflecting complementary. Then $f \in L^{2}(\Omega)$ is said 
to be compatible with the system if and only if there exist $U \in H^{1}\left(\Omega_{3} \backslash \Omega_{2}\right)$ and $V \in H^{1}\left(\Omega_{3} \backslash \Omega_{2}\right)$ such that

$$
\left\{\begin{array}{cl}
\operatorname{div}(A \nabla U)+k^{2} \Sigma U=F_{*} f-f & \text { in } \Omega_{3} \backslash \bar{\Omega}_{2}, \\
U=0 & \text { on } \partial \Omega_{2}, \\
A \nabla U \cdot \eta=0 & \text { on } \partial \Omega_{2},
\end{array}\right.
$$

and

$$
\left\{\begin{array}{cl}
\operatorname{div}(A \nabla V)+k^{2} \Sigma V=f & \text { in } \Omega_{3} \backslash \bar{\Omega}_{2}, \\
V=\left.W\right|_{\text {ext }} & \text { on } \partial \Omega_{3}, \\
A \nabla V \cdot \eta=\left.A \nabla W \cdot \eta\right|_{\text {ext }} & \text { on } \partial \Omega_{3} .
\end{array}\right.
$$

Here $W \in H^{1}\left(\Omega \backslash \partial \Omega_{3}\right)$ is the unique solution to the system

$$
\left\{\begin{array}{rlrl}
\operatorname{div}(\hat{A} \nabla W)+k^{2} \hat{\Sigma} W=\hat{f} & & \text { in } \Omega \backslash \partial \Omega_{3}, \\
W & =0 & & \text { on } \partial \Omega, \\
{[W]=-U} & & \text { on } \partial \Omega_{3}, \\
{[\hat{A} \nabla W \cdot \eta]} & =-A \nabla U \cdot \eta & & \text { on } \partial \Omega_{3} .
\end{array}\right.
$$

The compatibility condition is an intrinsic one, i.e., it does not depend on the choice of $F$ and $G$. In fact, it is equivalent to the boundedness of $\left\|u_{\delta}\right\|_{H^{1}(\Omega)}$ as $\delta \rightarrow 0$. Given $F$, there are infinitely many choices of $G$. A choice of $G$ that would make the compatibility condition more accessible is preferred. Problems (3.12) and (3.13) are the two Cauchy problems obtained from the reflecting technique. The reflecting complementary condition is necessary so that $\left(\left\|u_{\delta}\right\|_{H^{1}(\Omega)}\right)$ explodes for some $f$ (see [6]).

The uniqueness of $U$ and $V$ follow from the unique continuation principle (see, e.g., [1, 17]). The existence and uniqueness of $W$ are standard and follow from Fredholm's theory (see, e.g., 4]) since system (3.7) is well-posed.

The next result is in the spirit of (1.2).

Theorem 2. Let $d=2,3, \delta>0, f \in L^{2}(\Omega)$ and let $u_{\delta} \in H_{0}^{1}(\Omega)$ be the unique solution to equation (2.14):

$$
\operatorname{div}\left(s_{\delta} A \nabla u_{\delta}\right)+k^{2} s_{0} \Sigma u_{\delta}=s_{0} f \text { in } \Omega .
$$

Assume that the media $(A, \Sigma)$ in $\Omega_{3} \backslash \Omega_{2}$ and $(-A,-\Sigma)$ in $\Omega_{2} \backslash \Omega_{1}$ are reflecting complementary and $f$ is compatible with the system. Then $\left(u_{\delta}\right)$ converges weakly to $N I(f)$ in $H^{1}(\Omega)$, where

$$
N I(f)=\left\{\begin{array}{cl}
W & \text { if } x \in \Omega \backslash \Omega_{3}, \\
V & \text { if } x \in \Omega_{3} \backslash \Omega_{2}, \\
(U+V) \circ F & \text { if } x \in \Omega_{2} \backslash \Omega_{1}, \\
W \circ G \circ F & \text { if } x \in \Omega_{1} .
\end{array}\right.
$$

Here $U, V$, and $W$ are given in Definition 2. 
If $f=0$ in $\Omega_{3}$, then $U=0$. In this case, the compatibility condition is equivalent to the existence of $V \in H^{1}\left(\Omega_{3} \backslash \bar{\Omega}_{2}\right)$ to the Cauchy problem

$$
\left\{\begin{array}{cl}
\operatorname{div}(A \nabla V)+k^{2} \Sigma V=0 & \text { in } \Omega_{3} \backslash \bar{\Omega}_{2}, \\
V=\left.W\right|_{\text {ext }} & \text { on } \partial \Omega_{3}, \\
A \nabla V \cdot \eta=\left.A \nabla W \cdot \eta\right|_{\text {ext }} & \text { on } \partial \Omega_{3},
\end{array}\right.
$$

where $W \in H_{0}^{1}(\Omega)$ is the unique solution to

$$
\operatorname{div}(\hat{A} \nabla W)+k^{2} \hat{\Sigma} W=f \text { in } \Omega .
$$

We have

Corollary 1. Let $d=2,3$ and $f \in L^{2}(\Omega)$ with $\operatorname{supp} f \cap \Omega_{3}=\varnothing$. Assume that the media $(A, \Sigma)$ in $\Omega_{3} \backslash \Omega_{2}$ and $(-A,-\Sigma)$ in $\Omega_{2} \backslash \Omega_{1}$ are reflecting complementary and there exists a solution $V \in H^{1}\left(\Omega_{3} \backslash \bar{\Omega}_{2}\right)$ to (3.16). Then $f$ is compatible to the system.

It follows from Corollary 1 that if supp $f \cap \Omega_{3}=\varnothing$ and if $G_{*} F_{*} A(x)=A(x)$ and $G_{*} F_{*} \Sigma(x)=\Sigma(x)$ for $x \in \Omega_{3} \backslash \bar{\Omega}_{2}$, then $V=W$. We obtain

Corollary 2. Let $d=2,3$ and $f \in L^{2}(\Omega)$ with $\operatorname{supp} f \cap \Omega_{3}=\varnothing$. Assume that the media $(A, \Sigma)$ in $\Omega_{3} \backslash \Omega_{2}$ and $(-A,-\Sigma)$ in $\Omega_{2} \backslash \Omega_{1}$ are reflecting complementary and

$$
G_{*} F_{*} A(x)=A(x) \quad \text { and } \quad G_{*} F_{*} \Sigma(x)=\Sigma(x) \quad \text { for } \quad x \in \Omega_{3} \backslash \bar{\Omega}_{2} .
$$

Then $f$ is compatible to the system.

Remark 3 . It is easy to verify that the $2 \mathrm{~d}$ setting considered in the introduction satisfies the assumptions of Corollary 2 .

Remark 4. In this paper, we characterize the behavior of $u_{\delta}$ as $\delta \rightarrow 0$ for compatible $f$. The paper [10] develops the method introduced here to deal with this problem without the assumption on the compatibility in the cloaking setting.

3.2. Proofs of Theorems 1 and 2, This section containing two subsections is devoted to the proof of Theorems 1 and 2. In the first subsection, we establish basic properties of solutions to equation (2.14) such as existence, uniqueness, and stability, and establish a result on the change of variables concerning reflections. The proofs of Theorems 1 and 2 are given in the second subsection.

3.2.1. Preliminaries. This section contains two lemmas. The first one is on the well-posedness of (2.14).

Lemma 1. Let $d=2,3, k>0,0<\delta<1, g \in H^{-1}(\Omega)$ (the duality of $H_{0}^{1}(\Omega)$ ) and let $s_{\delta}$ be defined in (2.13). Assume that $A$ and $\Sigma$ satisfy (2.11) and (2.12), and (3.5) holds. Then there exists a unique solution $v_{\delta} \in H_{0}^{1}(\Omega)$ to the equation

$$
\operatorname{div}\left(s_{\delta} A \nabla v_{\delta}\right)+k^{2} s_{0} \Sigma v_{\delta}=g \text { in } \Omega .
$$

Moreover,

$$
\left\|v_{\delta}\right\|_{H^{1}(\Omega)} \leq C\left(\frac{1}{\delta}\|g\|_{H^{-1}(\Omega)}+\|g\|_{L^{2}\left(\Omega_{1}\right)}+\|g\|_{L^{2}\left(\Omega_{2} \backslash \bar{\Omega}_{1}\right)}+\|g\|_{L^{2}\left(\Omega \backslash \bar{\Omega}_{2}\right)}\right),
$$

for some positive constant $C$ independent of $g$ and $\delta$, as $\delta$ is small. 
Proof. The existence of $v_{\delta}$ follows from the uniqueness of $v_{\delta}$ by Fredholm's theorem (see, e.g., [4]). We now establish the uniqueness of $v_{\delta}$ by showing that $v_{\delta}=0$ if $v_{\delta} \in H_{0}^{1}(\Omega)$ is a solution to the equation

$$
\operatorname{div}\left(s_{\delta} A \nabla v_{\delta}\right)+k^{2} s_{0} \Sigma v_{\delta}=0 \text { in } \Omega .
$$

Multiplying the above equation by $\bar{v}_{\delta}$ (the conjugate of $v_{\delta}$ ) and integrating the obtained expression on $\Omega$, we have

$$
\int_{\Omega} s_{\delta}\left\langle A \nabla v_{\delta}, \nabla v_{\delta}\right\rangle d x-\int_{\Omega} k^{2} s_{0} \Sigma\left|v_{\delta}\right|^{2} d x=0 .
$$

This implies, by considering the imaginary part,

$$
\int_{\Omega_{2} \backslash \Omega_{1}}\left\langle A \nabla v_{\delta}, \nabla v_{\delta}\right\rangle d x=0 .
$$

It follows from (2.11) that $v_{\delta}$ is constant in $\Omega_{2} \backslash \Omega_{1}$. Thus $v_{\delta}=0$ in $\Omega_{2} \backslash \Omega_{1}$ since $\operatorname{div}\left(s_{\delta} A \nabla v_{\delta}\right)+k^{2} s_{0} \Sigma v_{\delta}=0$ in $\Omega_{2} \backslash \Omega_{1}$. This implies $v_{\delta}=0$ in $\Omega \backslash \Omega_{2}$ and in $\Omega_{1}$ by (3.5). Here to establish $v_{\delta}=0$ in $\Omega_{1}$, we considered the function $V_{\delta}$ defined in $\Omega$ as $V_{\delta}=v_{\delta} \circ F^{-1} \circ G^{-1}$ in $\Omega_{3}$ and $V_{\delta}=0$ in $\Omega \backslash \Omega_{3}$ and used the well-posedness of (3.7). The proof of the uniqueness is complete.

We next establish (3.19) by a contradiction argument. Assume that (3.19) is not true. Then there exists $\left(g_{\delta}\right) \subset H^{-1}(\Omega)$ such that

$$
\left\|v_{\delta}\right\|_{H^{1}(\Omega)}=1 \text { and } \frac{1}{\delta}\left\|g_{\delta}\right\|_{H^{-1}}+\left\|g_{\delta}\right\|_{L^{2}\left(\Omega_{1}\right)}+\left\|g_{\delta}\right\|_{L^{2}\left(\Omega_{2} \backslash \bar{\Omega}_{1}\right)}+\|g\|_{L^{2}\left(\Omega \backslash \bar{\Omega}_{2}\right)} \rightarrow 0,
$$

as $\delta \rightarrow 0$, where $v_{\delta} \in H_{0}^{1}(\Omega)$ is the unique solution to the equation

$$
\operatorname{div}\left(s_{\delta} A \nabla v_{\delta}\right)+k^{2} s_{0} \Sigma v_{\delta}=g_{\delta} \text { in } \Omega .
$$

Multiplying this equation by $\bar{v}_{\delta}$ and integrating the obtained expression on $\Omega$, we have

$$
\int_{\Omega} s_{\delta}\left\langle A \nabla v_{\delta}, \nabla v_{\delta}\right\rangle d x-\int_{\Omega} k^{2} s_{0} \Sigma\left|v_{\delta}\right|^{2} d x=-\int_{\Omega} g_{\delta} \bar{v}_{\delta} d x .
$$

Considering the imaginary part and using the fact that

$$
\frac{1}{\delta}\left|\int_{\Omega} g_{\delta} \bar{v}_{\delta}\right| \leq \frac{1}{\delta}\left\|g_{\delta}\right\|_{H^{-1}}\left\|v_{\delta}\right\|_{H^{1}(\Omega)} \rightarrow 0 \text { as } \delta \rightarrow 0 \text { by (3.20), }
$$

we obtain, by (2.11),

$$
\left\|\nabla v_{\delta}\right\|_{L^{2}\left(\Omega_{2} \backslash \Omega_{1}\right)} \rightarrow 0 \text { as } \delta \rightarrow 0 .
$$

Since $\operatorname{div}\left(A \nabla v_{\delta}\right)+k^{2} \Sigma v_{\delta}=g_{\delta}$ in $\Omega_{2} \backslash \bar{\Omega}_{1}$, it follows from (3.20) and a standard compactness argument that

$$
\left\|v_{\delta}\right\|_{L^{2}\left(\Omega_{2} \backslash \Omega_{1}\right)} \rightarrow 0 \text { as } \delta \rightarrow 0 .
$$

A combination of (3.22) and (3.23) yields

$$
\left\|v_{\delta}\right\|_{H^{1}\left(\Omega_{2} \backslash \Omega_{1}\right)} \rightarrow 0 \text { as } \delta \rightarrow 0 .
$$

In particular,

$$
\left\|v_{\delta}\right\|_{H^{1 / 2}\left(\partial \Omega_{2}\right)}+\left\|v_{\delta}\right\|_{H^{1 / 2}\left(\partial \Omega_{1}\right)} \rightarrow 0 \text { as } \delta \rightarrow 0 .
$$

We derive from the well-posedness of (3.6) and (3.7) that

$$
\left\|v_{\delta}\right\|_{H^{1}\left(\Omega \backslash \Omega_{2}\right)} \rightarrow 0
$$


and

$$
\left\|v_{\delta}\right\|_{H^{1}\left(\Omega \backslash \Omega_{1}\right)} \rightarrow 0 .
$$

A combination of (3.24), (3.25), and (3.26) yields

$$
\left\|v_{\delta}\right\|_{H^{1}(\Omega)} \rightarrow 0 \text {. }
$$

We have a contradiction by (3.20). The proof of (3.19) is complete.

The second lemma is on the change of variables for reflections.

Lemma 2. Let $k \geq 0$, let $D_{1}$ and $D_{2}$ be two smooth open subsets of $\mathbb{R}^{d}$, let $T$ be a diffeomorphism from $D_{1}$ onto $D_{2}$, let $a \in\left[L^{\infty}\left(D_{1}\right)\right]^{d \times d}$ be a matrix function, and let $\sigma \in L^{\infty}\left(D_{1}\right)$ be a complex function. Fix $u \in H^{1}\left(D_{1}\right)$ and set $v=u \circ T^{-1}$. Then

$$
\operatorname{div}(a \nabla u)+k^{2} \sigma u=f \text { in } D_{1}
$$

iff

$$
\operatorname{div}\left(T_{*} a \nabla v\right)+k^{2} T_{*} \sigma v=T_{*} f \text { in } D_{2} .
$$

Assume that $\Gamma_{1}$ and $\Gamma_{2}$ are open subsets of $\partial D_{1}$ and $\partial D_{2}$ such that $\Gamma_{1}$ and $\Gamma_{2}$ are smooth, $\Gamma_{2}=T\left(\Gamma_{1}\right)$, and $\mathbf{T}:=\left.T\right|_{\Gamma_{1}} \Gamma_{1} \rightarrow \Gamma_{2}$ is a diffeomorphism 3 We have

$$
a \nabla u \cdot \eta_{1}=g_{1} \text { on } \Gamma_{1}
$$

iff

where 4

$$
T_{*} a \nabla v \cdot \eta_{2}=g_{2} \text { on } \Gamma_{2}
$$

$$
g_{2}(y)=g_{1}(x) /|\operatorname{det} \nabla \mathbf{T}(x)| \text { with } x=\mathbf{T}^{-1}(y) .
$$

Here $\eta_{1}$ and $\eta_{2}$ are the normal unit vectors on $\Gamma_{1}$ and $\Gamma_{2}$ directed to the exterior of $D_{1}$ and $D_{2}$. In particular, if $\Gamma_{1}=\Gamma_{2}, \mathbf{T}(x)=x$ on $\Gamma_{1}, D_{2} \cap D_{1}=\varnothing$, then we have

$$
T_{*} a \nabla v \cdot \eta_{1}=-a \nabla u \cdot \eta_{1} \text { on } \Gamma_{1}=\Gamma_{2} .
$$

Proof. Lemma 2 is a consequence of the change of variables. The first equivalence relation is known and can be proved by using the weak formula for $u$ and $v$. The second equivalence follows similarly. The details are left to the reader.

3.2.2. Proof of the first statement of Theorem 1 and Theorem 2 . In this section, $f$ is compatible. The proof is derived from the following steps:

Step 1. Let $v \in H_{0}^{1}(\Omega)$ be a solution to the equation

$$
\operatorname{div}\left(s_{0} A \nabla v\right)+k^{2} s_{0} \Sigma v=s_{0} f \text { in } \Omega .
$$

We prove that $v=N I(f)$.

Step 2. Define $u_{0}:=N I(f)$. We prove that $u_{0} \in H_{0}^{1}(\Omega)$ is a solution to the equation

$$
\operatorname{div}\left(s_{0} A \nabla u_{0}\right)+k^{2} s_{0} \Sigma u_{0}=s_{0} f \text { in } \Omega .
$$

Step 3. We prove that $\left(u_{\delta}\right)_{0<\delta<1}$ is bounded in $H^{1}(\Omega)$.

\footnotetext{
${ }^{3}$ We assume here that there is an extension of $T$ in a neighborhood of $\partial D_{1}$ (which is also called $T)$ such that it is a diffeomorphism.

${ }^{4}$ In the identity below, $\nabla \mathbf{T}$ stands for the gradient of a transformation from a $(d-1)$-manifold into a $(d-1)$-manifold, and $\operatorname{det} \nabla \mathbf{T}$ denotes the determinant of a $(d-1) \times(d-1)$-matrix.
} 
Step 4 . We prove that $\left(u_{\delta}\right)$ converges weakly in $H^{1}(\Omega)$ and strongly in $L^{2}(\Omega)$ to $u_{0}$ as $\delta$ goes to 0 .

It is clear that the proof of the first statement of Theorem 1 and Theorem 2 is complete after these four steps. We now process these steps.

Step 1. Assume that $v \in H_{0}^{1}(\Omega)$ is a solution to the equation

$$
\operatorname{div}\left(s_{0} A \nabla v\right)+k^{2} s_{0} \Sigma v=s_{0} f \text { in } \Omega .
$$

Set

$$
v_{1}=v \circ F^{-1} \text { in } \Omega_{4} \backslash \bar{\Omega}_{2}
$$

and

$$
\hat{s}_{0}=\left\{\begin{array}{cl}
1 & \text { if } x \in \Omega_{3} \backslash \Omega_{2}, \\
-1 & \text { if } x \in \Omega_{4} \backslash \Omega_{3} .
\end{array}\right.
$$

Then $v_{1} \in H^{1}\left(\Omega_{3} \backslash \bar{\Omega}_{2}\right) \cap H_{l o c}^{1}\left(\Omega_{4} \backslash \bar{\Omega}_{2}\right)$ and, by Lemma 2, $v_{1}$ satisfies the equation

$$
\operatorname{div}\left(\hat{s}_{0} F_{*} A \nabla v_{1}\right)+k^{2} \hat{s}_{0} F_{*} \Sigma v_{1}=\hat{s}_{0} F_{*} f \text { in } \Omega_{4} \backslash \bar{\Omega}_{2},
$$

and

$$
v_{1}=v \text { on } \partial \Omega_{2} \quad \text { and } \quad F_{*} A \nabla v_{1} \cdot \eta=\left.A \nabla v \cdot \eta\right|_{\text {ext }} \text { on } \partial \Omega_{2} .
$$

In the last identity, we use the facts that $F_{*} A \nabla v_{1} \cdot \eta=-\left.A \nabla v \cdot \eta\right|_{\text {int }}$ on $\partial \Omega_{2}$ by (3.27), and that $\left.A \nabla v \cdot \eta\right|_{\text {ext }}=-\left.A \nabla v \cdot \eta\right|_{\text {int }}$ on $\partial \Omega_{2}$ by the transmission condition on $\partial \Omega_{2}$. Define

$$
\mathbf{U}=v_{1}-v \text { in } \Omega_{3} \backslash \bar{\Omega}_{2} .
$$

Since $F_{*} A=A$ and $F_{*} \Sigma=\Sigma$ in $\Omega_{3} \backslash \bar{\Omega}_{2}$, it follows that

$$
\left\{\begin{array}{cl}
\operatorname{div}(A \nabla \mathbf{U})+k^{2} \Sigma \mathbf{U}=F_{*} f-f & \text { in } \Omega_{3} \backslash \bar{\Omega}_{2}, \\
\mathbf{U}=0 & \text { on } \partial \Omega_{2}, \\
A \nabla \mathbf{U} \cdot \eta=0 & \text { on } \partial \Omega_{2} .
\end{array}\right.
$$

Applying the unique continuation principle (see, e.g., [1,17]), from (3.12), we have

$$
\mathbf{U}=U \text { in } \Omega_{3} \backslash \bar{\Omega}_{2} .
$$

Define $v_{2}$ in $\Omega$ as

$$
v_{2}(x)=\left\{\begin{array}{cl}
v_{1} \circ G^{-1}(x) & \text { if } x \in \Omega_{3}, \\
v(x) & \text { if } x \in \Omega \backslash \Omega_{3} .
\end{array}\right.
$$

Using (2.18) and applying Lemma 2, we have

$$
\operatorname{div}\left(\hat{A} \nabla v_{2}\right)+k^{2} \hat{\Sigma} v_{2}=\hat{f} \text { in } \Omega \backslash \partial \Omega_{3},
$$

and, on $\partial \Omega_{3}$,

$$
\begin{aligned}
\left.\hat{A} \nabla v_{2} \cdot \eta\right|_{\mathrm{ext}}-\left.\hat{A} \nabla v_{2} \cdot \eta\right|_{\mathrm{int}} & =\left.A \nabla v \cdot \eta\right|_{\mathrm{ext}}+\left.F_{*} A \nabla v_{1} \cdot \eta\right|_{\mathrm{ext}} \quad(\text { by (13.27) }) \\
& =\left.A \nabla v \cdot \eta\right|_{\mathrm{ext}}-\left.F_{*} A \nabla v_{1} \cdot \eta\right|_{\mathrm{int}} \quad(\text { by (3.31) }) \\
& =\left.A \nabla v \cdot \eta\right|_{\mathrm{ext}}-\left.F_{*} A \nabla(v+\mathbf{U}) \cdot \eta\right|_{\mathrm{int}} \quad(\text { by (3.32) }) .
\end{aligned}
$$


Since $F_{*} A=A$ in $\Omega_{3} \backslash \bar{\Omega}_{2}$, it follows from (3.34) that

$$
\left.\hat{A} \nabla v_{2} \cdot \eta\right|_{\text {ext }}-\left.\hat{A} \nabla v_{2} \cdot \eta\right|_{\text {int }}=-\left.A \nabla U \cdot \eta\right|_{\text {int }} \text { on } \partial \Omega_{3} .
$$

Since $G(x)=x$ on $\partial \Omega_{3}$, we obtain, on $\partial \Omega_{3}$,

$$
\left.v_{2}\right|_{\text {ext }}-\left.v_{2}\right|_{\text {int }}=\left.v\right|_{\text {ext }}-\left.v_{1}\right|_{\text {ext }}=\left.v\right|_{\text {ext }}-\left.v_{1}\right|_{\text {int }}=\left.v\right|_{\text {ext }}-\left.(\mathbf{U}+v)\right|_{\text {int }}=-U
$$

by (3.34). Combining (3.36), (3.37), (3.38), and (3.14), and applying the unique continuation principle, we have

$$
v_{2}=W \text { in } \Omega \text {. }
$$

Since $\operatorname{div}(A \nabla v)+k^{2} \Sigma v=f$ in $\Omega_{3} \backslash \bar{\Omega}_{2}$, it follows from (3.35) and (3.39) that

$$
\begin{cases}\operatorname{div}(A \nabla v)+k^{2} \Sigma v=f & \text { in } \Omega_{3} \backslash \bar{\Omega}_{2}, \\ v=\left.W\right|_{\text {ext }} & \text { on } \partial \Omega_{3}, \\ \left.A \nabla v \cdot \eta\right|_{\text {int }}=\left.A \nabla W \cdot \eta\right|_{\text {ext }} & \text { on } \partial \Omega_{3} .\end{cases}
$$

By the unique continuation principle, it follows from (3.13) that

$$
v=V \text { in } \Omega_{3} \backslash \bar{\Omega}_{2} .
$$

We claim that

$$
v=\left\{\begin{array}{cl}
W & \text { in } \Omega \backslash \Omega_{3}, \\
V & \text { in } \Omega_{3} \backslash \Omega_{2}, \\
(V+U) \circ F & \text { in } \Omega_{2} \backslash \Omega_{1}, \\
W \circ G \circ F & \text { in } \Omega_{1} .
\end{array}\right.
$$

In fact, the statement $v=W$ in $\Omega \backslash \Omega_{3}$ is a consequence of (3.35) and (3.39); the statement $v=V$ in $\Omega_{3} \backslash \Omega_{2}$ follows from (3.40); the statement $v=(V+U) \circ F$ in $\Omega_{2} \backslash \Omega_{1}$ is a consequence of the fact $v_{1}=v+U=V+U$ in $\Omega_{3} \backslash \Omega_{2}$, and the statement $v=v_{1} \circ F$ in $\Omega_{2} \backslash \Omega_{1} ; v=W \circ G \circ F$ in $\Omega_{1}$ is a consequence of the definition of $v_{1}$ and $v_{2}$, and $v_{2}=W$ in $\Omega_{3}$. The claim is proved. Therefore,

$$
v=N I(f) \text { in } \Omega \text {. }
$$

The proof of Step 1 is complete.

Step 2. We claim that

$$
\operatorname{div}\left(A \nabla u_{0}\right)+k^{2} \Sigma u_{0}=f \text { in } \Omega \backslash\left(\partial \Omega_{3} \cup \partial \Omega_{2} \cup \partial \Omega_{1}\right),
$$

where $u_{0}:=N I(f)$. Indeed, it is just a consequence of the definition of $U, V$ and $W$ and the facts that $F_{*} A=A$ and $F_{*} \Sigma=\Sigma$ in $\Omega_{3} \backslash \Omega_{2}$.

It remains to verify

$$
\left[A \nabla u_{0}\right]=\left[u_{0}\right]=0 \text { on } \partial \Omega_{3}, \quad\left[s_{0} A \nabla u_{0}\right]=\left[u_{0}\right]=0 \text { on } \partial \Omega_{2},
$$

and

$$
\left[A \nabla u_{0}\right]=\left[u_{0}\right]=0 \text { on } \partial \Omega_{1} .
$$

From the definitions of $V$ in (3.13) and $N I(f)$ in (3.15), we have

$$
\left[A \nabla u_{0}\right]=\left[u_{0}\right]=0 \text { on } \partial \Omega_{3} .
$$


Since $U=0$ and $A \nabla U \cdot \eta=0$ on $\partial \Omega_{2}$, it follows from (3.27) that

$$
\left[s_{0} A \nabla u_{0}\right]=\left[u_{0}\right]=0 \text { on } \partial \Omega_{2} .
$$

From (3.14) and (3.27), we have

$$
\left[s_{0} A \nabla u_{0}\right]=\left[u_{0}\right]=0 \text { on } \partial \Omega_{1} .
$$

The proof of Step 2 is complete.

Step 3. Set

$$
v_{\delta}=u_{\delta}-u_{0} \text { in } \Omega
$$

We have, in $\Omega$,

$$
\operatorname{div}\left(s_{\delta} A \nabla v_{\delta}\right)+k^{2} s_{0} \Sigma v_{\delta}=\operatorname{div}\left(s_{\delta} A \nabla u_{\delta}\right)-\operatorname{div}\left(s_{\delta} A \nabla u_{0}\right)+k^{2} s_{0} \Sigma u_{\delta}-k^{2} s_{0} \Sigma u_{0} .
$$

This implies

$$
\operatorname{div}\left(s_{\delta} A \nabla v_{\delta}\right)+k^{2} s_{0} \Sigma v_{\delta}=\operatorname{div}\left[\left(s_{0}-s_{\delta}\right) A \nabla u_{0}\right] \text { in } \Omega .
$$

By Lemma 1, we have

$$
\left\|\nabla v_{\delta}\right\|_{L^{2}(\Omega)} \leq C\left\|\nabla u_{0}\right\|_{L^{2}(\Omega)},
$$

which yields, since $u_{\delta}=v_{\delta}+u_{0}$,

$$
\left\|\nabla u_{\delta}\right\|_{L^{2}(\Omega)} \leq C\left\|\nabla u_{0}\right\|_{L^{2}(\Omega)} .
$$

Since $u_{\delta} \in H_{0}^{1}(\Omega)$, by Poincaré's inequality, it follows that

$$
\left\|u_{\delta}\right\|_{H^{1}(\Omega)} \leq C\left\|\nabla u_{0}\right\|_{L^{2}(\Omega)} .
$$

Step 3 is complete.

Step 4. The conclusion of Step 4 follows from Step 3 and the facts that the limit of $u_{\delta}$ (up to a subsequence) satisfies (3.28) and (3.28) has a unique solution in $H_{0}^{1}(\Omega)$ by Steps 1 and 2 .

3.2.3. Proof of the second statement of Theorem 1. In this section $f$ is not compatible. We prove the second statement of Theorem 1 by contradiction. Assume that (3.11) is not true. Without loss of generality, there exists a bounded sequence $\left(u_{\delta}\right)$ in $H_{0}^{1}(\Omega)$ such that $u_{\delta}$ is the unique solution to the equation

$$
\operatorname{div}\left(s_{\delta} A \nabla u_{\delta}\right)+k^{2} s_{0} \Sigma u_{\delta}=s_{0} f \text { in } \Omega,
$$

and $u_{\delta}$ converges weakly to (some) $u \in H^{1}(\Omega)$ as $\delta \rightarrow 0$. It follows that $u \in H_{0}^{1}(\Omega)$ is a solution to the equation

$$
\operatorname{div}\left(s_{0} A \nabla u\right)+k^{2} s_{0} u=s_{0} f \text { in } \Omega .
$$

Define

$$
U=u \circ F^{-1}-u \text { in } \Omega_{3} \backslash \bar{\Omega}_{2} \quad \text { and } \quad V=u \text { in } \Omega_{3} \backslash \bar{\Omega}_{2} .
$$

As in Step 1 of Section 3.2.2 $U$ and $V$ satisfy (3.12) and (3.13) respectively. We have a contradiction since $f$ is not compatible with the system.

\section{ACKNOWLEDGMENT}

The author would like to thank Bob Kohn and Grame Milton for interesting discussions. 


\section{REFERENCES}

[1] Giovanni Alessandrini, Strong unique continuation for general elliptic equations in 2D, J. Math. Anal. Appl. 386 (2012), no. 2, 669-676, DOI 10.1016/j.jmaa.2011.08.029. MR2834777

[2] Habib Ammari, Giulio Ciraolo, Hyeonbae Kang, Hyundae Lee, and Graeme W. Milton, Spectral theory of a Neumann-Poincaré-type operator and analysis of cloaking due to anomalous localized resonance, Arch. Ration. Mech. Anal. 208 (2013), no. 2, 667-692, DOI 10.1007/s00205-012-0605-5. MR3035988

[3] Guy Bouchitté and Ben Schweizer, Cloaking of small objects by anomalous localized resonance, Quart. J. Mech. Appl. Math. 63 (2010), no. 4, 437-463, DOI 10.1093/qjmam/hbq008. MR2738456(2011k:35036)

[4] Haïm Brezis, Analyse fonctionnelle (French), Collection Mathématiques Appliquées pour la Maîtrise. [Collection of Applied Mathematics for the Master's Degree], Masson, Paris, 1983. Théorie et applications. [Theory and applications]. MR697382(85a:46001)

[5] O. P. Bruno and S. Lintner, Superlens-cloaking of small dielectric bodies in the quasistatic regime, J. Appl. Phys. 102 (2007), 12452.

[6] R. V. Kohn, J. Lu, B. Schweize, and M. I. Weinstein, A variational perspective on cloaking by anomalous localized resonance, (2013), http://arxiv.org/abs/1210.4823.

[7] Y. Lai, H. Chen, Z. Zhang, and C. T. Chan, Complementary media invisibility cloak that cloaks objects at a distance outside the cloaking shell, Phys. Rev. Lett. 102 (2009), 093901.

[8] Y. Lai, J. Ng, H. Chen, D. Han, J. Xiao, Z. Zhang, and C. T. Chan, Illusion optics: The optical transformation of an object into another object, Phys. Rev. Lett. 102 (2009), 253902.

[9] H. M. Nguyen and H. L. Nguyen, Complete resonance and localized resonance in plasmonic structures, (2013), http://arxiv.org/pdf/1310.3633.pdf.

[10] H. M. Nguyen, Cloaking using complementary media in the quasistatic regime, (2013), http://arxiv.org/pdf/1310.5483.pdf.

[11] Graeme W. Milton and Nicolae-Alexandru P. Nicorovici, On the cloaking effects associated with anomalous localized resonance, Proc. R. Soc. Lond. Ser. A Math. Phys. Eng. Sci. 462 (2006), no. 2074, 3027-3059, DOI 10.1098/rspa.2006.1715. MR2263683 (2008e:78018)

[12] G. W. Milton, N. P. Nicorovici, R. C. McPhedran, K. Cherednichenko, and Z. Jacob, Solutions in folded geometries, and associated cloaking due to anomalous resonance, New J. Phys. 10 (2008), 115021.

[13] G. W. Milton, N. P. Nicorovici, R. C. McPhedran, and V. A. Podolskiy, A proof of superlensing in the quasistatic regime, and limitations of superlenses in this regime due to anomalous localized resonance, Proc. R. Soc. Lond. Ser. A Math. Phys. Eng. Sci. 461 (2005), no. 2064, 3999-4034, DOI 10.1098/rspa.2005.1570. MR2186014 (2006h:78001)

[14] N. A. Nicorovici, R. C. McPhedran, and G. W. Milton, Optical and dielectric properties of partially resonant composites, Phys. Rev. B 49 (1994), 8479-8482.

[15] J. B. Pendry, Negative refraction makes a perfect lens, Phys. Rev. Lett. 85 (2000), 3966-3969.

[16] _ Perfect cylindrical lenses, Optics Express 1 (2003), 755-760.

[17] M. H. Protter, Unique continuation for elliptic equations, Trans. Amer. Math. Soc. 95 (1960), 81-91. MR0113030(22 \#3871)

[18] S. A. Ramakrishna and J. B. Pendry, Focusing light using negative refraction, J. Phys.: Condens. Matter 15 (2003), 6345.

[19] _ Spherical perfect lens: Solutions of Maxwell's equations for spherical geometry, Phys. Rev. B 69 (2004), 115115.

[20] R. A. Shelby, D. R. Smith, and S. Schultz, Experimental verification of a negative index of refraction, Science 292 (2001), 77-79.

[21] D. R. Smith, W. J. Padilla, D. C. Vier, S. C. Nemat-Nasser, and S. Schultz, Composite medium with simultaneously negative permeability and permittivity, Phys. Rev. Lett. 84 (2000), 41844187.

[22] V. G. Veselago, The electrodynamics of substances with simultaneously negative values of $\varepsilon$ and $\mu$, Usp. Fiz. Nauk 92 (1964), 517-526.

Chair of Analysis and Applied Mathematics, École Polytechnique Féderale de LauSanne, Station 8, CH-1015 Lausanne, Switzerland - School of Mathematics, University of Minnesota, Minneapolis, Minnesota 55455

E-mail address: hoai-minh.nguyen@epfl.ch

E-mail address: hmnguyen@math.umn.edu 